Enferm Bras 2020;19(2):154-62

https://doi.org/10.33233/eb.v19i2.3640

\title{
ARTIGO ORIGINAL \\ Percepção da equipe de enfermagem da Estratégia de Saúde da Família quanto aos cuidados paliativos
}

Nayanne Ferreira Geralda Rezende*, Meiriane Nogueira Garcia*, Alexandre Ernesto Silva, D.Sc. ${ }^{\star *}$, Fernanda Marcelino de Rezende e Silva, M.Sc. ${ }^{* \star}$, Silmara Nunes Andrade, D.Sc. ${ }^{* \star \star *}$, Flávia de Oliveira, D.Sc. ${ }^{\star \star \star \star *}$, Karla Amaral Nogueira Quadros ${ }^{\star \star \star \star \star \star}$

*Enfermeira, Universidade do Estado de Minas Gerais, unidade Divinópolis, "*Enfermeiro, Professor Adjunto da Universidade Federal de São João Del-Rei, ${ }^{\star * *}$ Enfermeira, Coordenadora e docente do curso de enfermagem da Universidade do Estado de Minas Gerais, unidade Divinópolis, ${ }^{* * * \star}$ Enfermeira, Docente do Departamento de Enfermagem da Universidade do Estado de Minas Gerais, unidade Divinópolis, ${ }^{* * * * *}$ Enfermeira, Professora Adjunta da Universidade Federal de São João Del-Rei, ${ }^{* * * \star * \star}$ Enfermeira, Doutoranda em ciências da saúde pela Universidade Federal de São João Del-Rei, Docente da Universidade do Estado de Minas Gerais, unidade Divinópolis

Recebido em 9 de dezembro de 2019; aceito em 22 de abril de 2020.

Correspondência: Meiriane Nogueira Garcia, Universidade do Estado de Minas Gerais, Rua Presidente Tancredo Neves, 417, 35555-000 Carmo do Cajuru MG

Artigo extraído do trabalho de conclusão de curso intitulado: Percepção da equipe de enfermagem da estratégia de saúde da família quanto aos cuidados paliativos. Universidade do Estado de Minas Gerais, 2018.

Nayanne Ferreira Geralda Rezende: rezendenayanne@gmail.com

Meiriane Nogueira Garcia: meirianeng@gmail.com

Alexandre Ernesto Silva: alexandresilva@ufsj.edu.br

Fernanda Marcelino de Rezende e Silva: fernanda.silva@uemg.br

Silmara Nunes Andrade: silmaranunesandrade@hotmail.com

Flávia de Oliveira: flaviadeoliveira@ufsj.edu.br

Karla Amaral Nogueira Quadros: kanq@bol.com.br

\section{Resumo}

Introdução: Os cuidados paliativos têm como foco a melhoria da qualidade de vida do paciente e seus familiares mediante uma patologia que ameace a vida. Percebe-se que geralmente os cuidados paliativos não são considerados como deveriam, existem barreiras na assistência dos cuidados paliativos na Estratégia de Saúde da Família (ESF). Dessa forma, este estudo tem como objetivo analisar qualitativamente os cuidados dispensados por profissionais de enfermagem às pessoas elegíveis ao cuidado paliativo nas Estratégias de Saúde da Família de um município do Centro-Oeste Mineiro. Métodos: Trata-se de uma pesquisa qualitativa realizada na cidade de Divinópolis/MG. A coleta de dados foi realizada por meio de entrevista guiada por roteiro semiestruturado realizada com 10 equipes de ESF sorteadas previamente, até a ocorrência de saturação dos dados. Resultados: Após transcrição, os conteúdos foram analisados emergindo assim três categorias: definindo cuidados paliativos na perspectiva de profissionais da enfermagem da Estratégia de Saúde da Família; a prestação de cuidados paliativos na Estratégia de Saúde da família, atitudes práticas; desafios para a execução dos cuidados paliativos na Estratégia de Saúde da Família. Conclusão: Espera-se com este estudo, contribuir como alerta para que os profissionais busquem qualificação e aperfeiçoamento para o desenvolvimento dos cuidados paliativos, bem como aguçar os gestores para a realização de educação em cuidados paliativos destinados à sua equipe.

Palavras-chave: Enfermagem, cuidados paliativos, doente terminal, percepção, cuidados de enfermagem.

\section{Abstract}


Perception of palliative care in the nursing team of the Family Health Strategy Introduction: Palliative care focuses on improving the quality of life of patients and their families through a pathology that threatens life. It is noticed that palliative care is generally not considered as it should be, there are barriers in palliative care assistance in the Family Health Strategy. This study aims to qualitatively analyze the care provided by nursing professionals to people eligible for palliative care in the Family Health Strategies of a city in the Midwest of Minas Gerais. Methods: This is a qualitative research carried out in the city of Divinópolis/MG. Data collection was carried out by means of an interview guided by a semi-structured script carried out with $10 \mathrm{FHS}$ teams drawn previously, until the occurrence of data saturation. Results: After transcription, the contents were analyzed thus emerging three categories: defining palliative care from the perspective of nursing professionals in the Family Health Strategy; the provision of palliative care in the Family Health Strategy, practical attitudes; challenges for the implementation of palliative care in the Family Health Strategy. Conclusion: This study is expected to contribute as an alert for professionals to seek qualification and improvement for the development of palliative care, as well as to sharpen managers to carry out education in palliative care for their team.

Keywords: Nursing, palliative care, terminally ill, perception, nursing care.

\section{Resumen \\ Percepción del equipo de enfermería de la Estrategia de Salud Familiar en cuanto al cuidado paliativo}

Introducción: Los cuidados paliativos se centran en mejorar la calidad de vida de los pacientes y sus familias a través de una patología que amenaza la vida. Se observa que los cuidados paliativos generalmente no se consideran como deberían ser, hay barreras en la asistencia de cuidados paliativos en la Estrategia de Salud Familiar. Por lo tanto, este estudio tiene como objetivo analizar cualitativamente la atención brindada por profesionales de enfermería a personas elegibles para cuidados paliativos en las Estrategias de Salud Familiar de una ciudad en el medio oeste de Minas Gerais. Métodos: Esta es una investigación cualitativa realizada en la ciudad de Divinópolis/MG. La recopilación de datos se realizó mediante una entrevista guiada por un guión semiestructurado realizado con 10 equipos de FHS elaborados previamente, hasta la aparición de saturación de datos. Resultados: Después de la transcripción, se analizaron los contenidos, surgiendo así tres categorías: definición de cuidados paliativos desde la perspectiva de los profesionales de enfermería en la Estrategia de Salud Familiar; la provisión de cuidados paliativos en la Estrategia de Salud Familiar, actitudes prácticas; desafíos para la implementación de los cuidados paliativos en la Estrategia de salud familiar. Conclusión: Se espera que este estudio contribuya como una alerta para que los profesionales busquen la calificación y la mejora para el desarrollo de los cuidados paliativos, así como para agudizar a los gerentes para llevar a cabo la educación en cuidados paliativos para su equipo.

Palabras-clave: Enfermería, cuidados paliativos, enfermedad terminal, percepción, cuidados de enfermería.

Introdução

Com a crescente disseminação do cristianismo pela Europa, há relatos a partir do século $\mathrm{V}$, da criação de abrigos e instituições de caridade, com intuito de cuidar dos peregrinos, e mais tarde destinar o cuidado a doentes, órfãos e pobres. Estes locais chamavam hospice, que significava hospedaria, assim surgiu historicamente o termo que designaria os cuidados paliativos (CP). Estes cuidados eram realizados por leigos, muitos destes ligados a entidades religiosas baseados na caridade. A partir de 1947, a inglesa, Dame Cicely Saunders, enfermeira, médica, iniciou estudos para minimizar a dor nos pacientes em fase final de vida, tornando-se uma das grandes defensoras dos cuidados prestados no final da vida [1-3].

No ano de 1967, o movimento de Hospice Moderno foi iniciado e, além da assistência aos doentes, houve o incentivo às atividades de ensino e pesquisa em todo o mundo. Assim, o Cuidado Paliativo moderno inclui o primeiro estudo sistemático com mais de 1.000 pacientes com diagnóstico de câncer em estágio avançado no St. Joseph's Hospice durante o período de 1958 e 1965 [1-3].

A partir de 1982, o Comitê de Câncer da Organização Mundial de Saúde (OMS) instituiu grupos para determinar políticas de hospice, com o intuito do alívio da dor e sofrimento de pessoas em fase final de vida. Posteriormente a OMS aderiu ao termo CP e anos depois foi publicada a primeira definição $[1,3]$. Em 2002, a OMS definiu os CP como: 
"cuidados paliativos consistem na assistência promovida por uma equipe multidisciplinar, que objetiva a melhoria da qualidade de vida do paciente e seus familiares, diante de uma doença que ameace a vida, por meio da prevenção e alívio do sofrimento, da identificação precoce, avaliação impecável e tratamento de dor e demais sintomas físicos, sociais, psicológicos e espirituais" [4:1].

Os CP são norteados por princípios descritos pela OMS no ano de 2002, que apresentam probabilidades diversas e intervenções clínicas. Dentre estas estão: promoção do alívio da dor e de outros sintomas estressantes; reafirmar a vida e ver a morte como um problema natural; a não pretensão em antecipar e nem postergar a morte; integrar aspectos psicossociais e espirituais ao cuidado; oferecer um sistema de suporte que auxilia a pessoa a viver tão ativamente quanto possível, até sua morte; oferecer um sistema de suporte que auxilia a família e entes queridos a sentir-se amparados durante todo o processo da doença; iniciar o mais precocemente possível, junto a outras medidas de prolongamento de vida, como a quimioterapia e radioterapia, e incluir todas as investigações necessárias para melhor compreensão e manejo dos sintomas [3,5]. No Brasil os CP tiveram início entre os anos de 1980, no estado do Rio Grande do Sul, com expansão de diversos serviços. Em 1997 foi criada a Associação Brasileira de Cuidados Paliativos (ABCP), para assim apoiar a formação de profissionais capacitados para esta temática. Objetivando a atuação destes profissionais nesta área, foi fundada no ano de 2005 , por um grupo de médicos, a Academia Nacional de Cuidados Paliativos (ANCP) [2].

$\mathrm{O}$ desenvolvimento dos $\mathrm{CP}$ abrange tanto os âmbitos ambulatoriais quanto os hospitalares e domiciliares. O tratamento e acompanhamento de pessoas devem ser realizados pela Estratégia de Saúde da Família (ESF), no próprio domicílio do indivíduo, com o intuito de diminuir custos com internações hospitalares prolongadas e promover contato entre a família e a pessoa, preservando sua integridade física e psicológica, além de proporcionar um cuidado humanizado [6].

A ESF foi implantada no Brasil, em 1994, com o objetivo de estender à população brasileira o acesso à saúde, conforme as diretrizes e princípios do Sistema Único de Saúde (SUS). A pessoa deve entrar no sistema através da unidade básica de saúde, que é representada pela ESF e centros de saúde, locais onde há uma relação da população adscrita no seu território de abrangência com a equipe de saúde. Desta forma, a unidade é responsável pelo cuidado, orientação, promoção à saúde, prevenção de doenças, redução dos agravos, reabilitação e tratamento do paciente através de uma equipe multidisciplinar [7].

$\mathrm{Na}$ vivência profissional e acadêmica, percebemos que geralmente os $\mathrm{CP}$ não são considerados como deveriam. A equipe encontra barreiras no que diz respeito ao binômio pessoa-família, pois muitos acreditam que os CP devem ser destinados somente as pessoas e os familiares que são carentes quanto a este apoio. Daí a importância da análise deste cuidado na ESF, para trazer aos familiares e indivíduos um conforto perante a morte [2].

O CP é prestado a pessoas portadoras de doenças ou agravos, com o intuito de minimizar o sofrimento destas pessoas e de seus familiares, frente à fase de adoecer e morrer. Diante disto parte a questão: como este cuidado é realizado pela equipe de enfermagem e qual a percepção desta frente a esses processos? Desse modo, o estudo teve como objetivo: analisar o cuidado às pessoas em CP nas ESF's, do município de Divinópolis/MG, utilizando como referência os princípios que orientam esta prática.

\section{Material e métodos}

Trata-se de uma pesquisa qualitativa. O estudo qualitativo faz uma relação entre os significados de valores, crenças, atitudes ou motivos presentes na sociedade, com o intuito de dar sentido aos dados coletados. Assim o ser humano possui diferenças de pensamentos, no modo de agir e de acordo com a sua realidade possui determinados comportamentos que são partilhados em sociedade $[8,9]$.

A pesquisa foi realizada na cidade de Divinópolis/MG, com os profissionais de enfermagem das ESF. O município está localizado no Centro-Oeste Mineiro, conta com uma população estimada de 234.937 habitantes [10].

O estudo foi realizado com 10 equipes de ESF sorteadas previamente, até a ocorrência de saturação dos dados. Participaram do estudo 24 profissionais de enfermagem vinculados às ESF do município de Divinópolis, ou seja, enfermeiro, enfermeiro residente e técnico de enfermagem. Durante a coleta de dados dois participantes não quiseram participar do estudo. 
As entrevistas foram realizadas nos meses de outubro e novembro de 2017. Inicialmente o próprio entrevistador entrou em contato com o responsável pela EFS, via telefone, abordando os profissionais de forma direta, para explicações quanto às propostas e objetivos da pesquisa, e foram agendadas as visitas para submissão dos roteiros da entrevista. A visita ocorreu nos dias determinados pelos profissionais, com duração de cerca de três horas em cada unidade, ocorrendo em dias distintos entre elas, de acordo com a disponibilidade de cada unidade/profissional.

Foi aplicado individualmente um roteiro de entrevista com as informações dos métodos de cuidados realizados em pessoas elegíveis aos cuidados paliativos, questões relacionadas ao conhecimento do cuidado paliativo, vivência profissional, profissão, tempo de graduação, idade, gênero, tempo na ESF, experiências laborais anteriores, que foi respondido pelos profissionais, em uma sala que estava disponível no momento da entrevista, dentro da própria unidade. Posteriormente a análise das entrevistas teve início com digitação na íntegra conforme escrito pelos profissionais, estes foram identificados por siglas P1 (profissional 1...), assim por diante.

Foi utilizado como critérios de inclusão: ser profissional de enfermagem, que estivessem vinculados nas ESF e assinar o Termo de Consentimento Livre e Esclarecido (TCLE). Os critérios de exclusão foram: estar gozando férias ou licença no momento da pesquisa.

As entrevistas foram digitadas no Word, e trechos das respostas da equipe colocados no texto conforme descrito nos roteiros. Posteriormente foi realizada a análise deste conteúdo sob a perspectiva de Bardin, esta consiste em uma análise de um conjunto de técnicas das respostas individuais e diversificadas dos profissionais, com o intuito de sistematizar o conhecimento geral da equipe [11].

O estudo passou pelo comitê de Ética da Instituição conforme disposto na resolução no466/2012 do Conselho Nacional de Saúde, e a confidencialidade das informações foram preservadas e respeitadas, através do parecer $n^{0}$ 2.342.319. Os gastos referentes à pesquisa foram de total responsabilidade dos pesquisadores.

Resultados

Os entrevistados foram em sua maioria do sexo feminino, destes 23 profissionais $(88,46 \%)$ eram mulheres, em sua pluralidade os trabalhadores tinham idade entre 23 e 53 anos, o tempo de graduação variou de oito meses a 20 anos de formados, foram entrevistados 15 enfermeiros e 11 técnicos de enfermagem.

Posteriormente a transcrição das entrevistas e análise emergiram três categorias: Definindo CP na perspectiva de profissionais da enfermagem da ESF; a prestação de CP na ESF, atitudes práticas; desafios para a execução dos CP na ESF.

\section{Definindo CP na perspectiva de profissionais da enfermagem da ESF}

Identificou-se que prevalece em todas as falas enquanto definição de CP o oferecimento de conforto e o alívio da dor às pessoas com prognósticos ruins. Seguem abaixo algumas falas que explicitam o exposto.

Cuidados para o bem-estar e conforto do paciente visando uma melhoria para sua qualidade de vida. (P17)

Cuidado paliativo seria oferecer o conforto, a paz, através de ações que proporcionem uma melhor "aceitação" do problema, do momento enfrentado[...]. (P26)

São cuidados como para aliviar a dor, quando não há outra forma de tratar a patologia - tratar os sintomas. (P7)

Cuidados paliativos são atividades/cuidados que podemos ter para que 0 paciente tenha um bom final (alívio da dor, fazer o que o paciente gostava de fazer, mantê-lo perto da família). Já ouvi falar muito em cursos e treinamentos, e já li sobre o assunto. (P11) 
São cuidados que o profissional tem com aquele paciente com alguma doença que tenha prognóstico ruim (como doença crônico degenerativa, câncer terminal), com objetivo de aliviar a dor, favorecer a dignidade humana, aliviar outros sintomas que o paciente tenha, verificar sonhos/desejos. (P1)

O cuidado paliativo é um método de cuidado realizado para doenças crônicas sem prognóstico de cura. É realizado principalmente na fase final de vida de modo a promover alívio dos sintomas físicos e sofrimento da alma[...]. (P12)

\section{A prestação de $C P$ na $E S F$, atitudes práticas}

Avaliando as condutas profissionais para prestação dos CP, identificou-se em $83,3 \%$ das falas que há ausência de sistematização quanto às práticas de CP na ESF. Além disso, os profissionais buscam orientar as pessoas elegíveis aos CP e os familiares quanto a realização da assistência, assim, há nas unidades uma discussão individual de cada caso com a equipe multidisciplinar para avaliar a conduta mais adequada a cada pessoa. As principais falas dessa categoria foram selecionadas para demonstrar o exposto.

Atualmente não existe nada sistematizado sobre cuidados paliativos na ESF. Quando tem algum paciente, discutimos o caso e condutas com o médico, porém assistemático. (P1)

Em minha prática diária o cuidado paliativo é oferecido sem sistematização, portanto não há uma pessoa específica, tentamos eleger a pessoa mais próxima daquele paciente. (P26)

É realizado cuidado pela equipe de enfermagem e médica como curativo, medicação, avaliação da fisioterapia e psicologia. Orientação a família sobre cuidados. A família procura a unidade ou o ACS traz a demanda para equipe, é feita visita de um profissional de nível superior da equipe, médico ou enfermeiro o paciente é avaliado, levantado suas necessidades e quais profissionais da equipe realizarão, também pode ser solicitado avaliação de outros profissionais como fisioterapeuta e psicólogo e encaminhamento para atenção secundária se necessário. A família é orientada quanto ao seu papel no cuidado. (P23)

É feita a discussão do caso de cada paciente. A equipe de enfermagem vai à residência conversa com a família e/ou cuidadores sobre quem irá realizar os cuidados e então treinamos e orientamos. Fazemos a supervisão de acordo com cada situação. (P8)

O paciente em cuidados paliativos é contra-referenciado por outro serviço de saúde a ESF, onde a equipe multidisciplinar trabalha em função, a fim de promover qualidade de vida a esse paciente. (P3

A equipe multidisciplinar irá discutir o caso, após detectar que o paciente não tem perspectiva de cura, possui uma doença que ameace a vida seguindo os critérios do manual da ANCP. A equipe multidisciplinar: médico, enfermeiro, técnico de enfermagem, psicólogo, assistente social, nutricionista. (P6)

\section{Desafios para a execução dos CP na ESF}

Quanto aos desafios observou-se falta de conhecimento sobre CP, bem como insuficiência numérica de profissionais para prestação dos CP. Notou-se que a grande maioria deles, ou seja, $16(61,54 \%)$ não obtiveram em sua trajetória profissional, formação e capacitação em CP, nove $(34,61 \%)$ dos trabalhadores tiveram durante graduação/pós graduação, disciplina optativa com esta temática e um destes $(3,85 \%)$ relatou a participação em Liga Acadêmica de CP na instituição de ensino. 
Falta de conhecimento por parte da equipe (conhecimento científico), treinamento. Tentamos sempre discutir casos e eu pessoalmente tento ler sobre $o$ assunto. (P11)

Os desafios se iniciam diante conhecimento, entendimento dos profissionais e mudança de comportamento, em aceitar a morte de quem é tratado; saber identificar os pacientes e abordar a família neste momento que requer empatia e delicadeza. Além de proporcionar cuidados nos quais o paciente se sinta confortável e for da sua vontade. (P25)

Os desafios são equipe incompletas (Falta ACS), demanda espontânea alta, modelo curativista, não tem equipe de Atendimento Domiciliar no município (mesmo estando dentro dos critérios para receber essas equipes). Os pacientes elegíveis para CP precisam ser acompanhados mais de perto, porém a demanda espontânea alta e outros não permite esse acompanhamento. Aos poucos, estamos procurando reorganizar o processo de trabalho da ESF. (P1)

Um dos desafios é a equipe reduzida, e o grande número de atribuições da enfermeira, e para superar isto tentamos trabalhar com as prioridades. (P23)

Em minha formação não realizei nenhum tipo de formação ou capacitação em cuidados paliativos, somente conhecimento acadêmico sobre $o$ assunto. (P3)

Não realizei treinamento. Apenas estudos próprios. (P21)

Não existe capacitação aos profissionais da ESF. Falta governabilidade e é necessário implantar equipes de atenção domiciliar no município. A equipe tem se programado a dar suporte aos usuários que necessitam ou que são elegíveis a CP com programação dos atendimentos interdisciplinares. (P12)

Acho que o maior desafio neste aspecto são as capacitações e geralmente as unidades estão sempre com muita demanda espontânea por isso esse cuidado paliativo as vezes fica comprometido, porém aqui nesta ESF percebo uma equipe muito comprometida que faz o seu melhor e cada dia supera muito bem esses problemas da demanda e consegue atender muito bem a população. (P10)

Sim, a disciplina optativa de cuidados paliativos da UFSJ. Contribui para uma nova visão do paciente em $C P$, na forma de cuidar e agir para melhorar o conforto do paciente nos momentos finais de vida, proporcionando uma morte digna. Através da residência em saúde da família/atenção básica que também tem a disciplina de cuidados paliativos. (P6)

Estou cursando uma disciplina na pós-graduação e está me acrescentando muito, pois temos pacientes em cuidados paliativos. (P2)

Participei de uma liga de cuidados paliativos na graduação. E atualmente faço uma disciplina de cuidados paliativos na residência de enfermagem em atenção básica. (P18) 
Quanto às definições de $\mathrm{CP}$ relatadas pelos profissionais, foram identificadas palavras chaves quanto a este significado, tais como: o conforto, este é definido como: "Conforto etimologicamente, se origina do latim confortar e, que significa fortificar, certificar, corroborar, conceder, consolar, aliviar, assistir, ajudar e auxiliar. O conforto pode assumir o significado de ato de confortar a si e de confortar o outro" [12:533].

Outro ponto citado foi o alívio da dor, que pode ser observado em outros estudos, como um indicativo de melhora da condição de vida das pessoas que se encontram em CP, com o intuito de diminuir a dor e o sofrimento causado durante o desenvolvimento da doença ou no decorrer do tratamento $[13,14]$.

O tratamento não envolve somente procedimentos e medicamentos, pois os indivíduos desenvolviam técnicas individuais para alívio da dor, como, por exemplo, ficar ao lado de familiares e amigos, pedir ao profissional que conversasse com ele durante o procedimento, posições indicadas para os procedimentos, além da cooperação quando estes fossem realizados [15].

Esta pesquisa teve coerência com a definição exposta pelos profissionais em outro estudo, no qual define o CP como um prognóstico ruim, demonstrando que o profissional é treinado para salvar vidas, seguindo um modelo curativista e não para aliviar o sofrimento dela diante de um quadro de doença que seja incompatível com a vida, assim o profissional pode se sentir frustrado mediante este processo [16].

Com a prestação dos CP nas unidades de ESF, observamos que muitos profissionais mencionaram a ausência de sistematização da assistência, como um entrave na realização de ações práticas para a execução destes cuidados, o que também foi comprovado em outro estudo [17].

A sistematização da assistência é uma atividade privativa do enfermeiro, que deve ser realizada tanto na rede pública e privada de saúde, conforme disposto na Resolução do COFEN no 358/2009. Este profissional deve utilizá-la com o intuito de organização do ambiente de trabalho, este processo se organiza através das seguintes etapas: coleta de dados de Enfermagem, diagnóstico de Enfermagem, planejamento de Enfermagem, implementação e avaliação de Enfermagem [18].

Um segundo tópico citado foi quanto à orientação, neste caso observa-se a necessidade que esta seja feita tanto com a pessoa quanto com seus familiares, para que estes consigam cuidar do indivíduo quando o profissional não estiver no seu domicílio. A interação entre o trinômio, equipe-pessoas-familiares, pode propiciar uma melhor aceitação da doença, bem como a adesão ao tratamento e preparo para a terminalidade da vida [19].

Além disso, foi notado que os profissionais realizam reuniões para discussão individual dos casos para definir qual a melhor terapêutica indicada para estes indivíduos. Neste tipo de conduta de abordagem multiprofissional, cada profissional tem sua contribuição para beneficiar o paliativismo, promovendo assim uma assistência humanizada e integralizada na tentativa de diminuir o sofrimento diante ao processo de morte [13,20].

Nota-se que as ESF contam com profissionais em diversas áreas de atuação, o que beneficia os cuidados, pois a pessoa é vista de maneira holística, e seu problema físico, social e emocional é tratado, para tentar diminuir o sofrimento deste período difícil. Porém esta gama de profissionais não se encontra na unidade em tempo integral, podendo originar dificuldades para prestação da assistência.

Os maiores desafios encontrados pelos profissionais para a realização dos CP foram a falta de conhecimento por parte dos profissionais quanto a esta temática, bem como a carência quanto à formação e capacitação durante sua trajetória acadêmica, o que sugere mais pesquisas voltadas ao CP [21]. Podendo estar correlacionado com o fato do profissional se acomodar com a rotina intensa de trabalho, e não buscar aprimoramentos que aperfeiçoem seu trabalho, podendo prejudicar a assistência, pois quando não há domínio do tema, torna-se mais difícil a realização dos cuidados, não é possível tratar o paciente de maneira sistematizada, tornando a assistência inadequada.

No que tange a insuficiência de profissionais, fato este pode ser observado tanto na equipe de enfermagem quanto nos demais membros da ESF, podendo gerar uma sobrecarga de funções práticas e administrativas que dificultam que o profissional exerça a assistência em CP [21].

Por conseguinte, notou-se que uma parcela da amostra realizou durante a graduação ou pós-graduação disciplina optativa em $\mathrm{CP}$, e uma integrante relatou a participação na Liga Acadêmica de CP da instituição, o que é de grande valia para a formação do profissional, que 
pode desenvolver na futura unidade de trabalho ações voltadas a este público, bem como capacitar sua equipe.

Durante a trajetória acadêmica deparamos com instituições de ensino que algumas vezes não enfocam no $\mathrm{CP}$. O estudante é preparado para exercício da enfermagem, porém disciplinas pontuais muitas vezes não são realizadas, tendo o estudante que buscar por conta própria conhecimentos mais abrangentes para conseguir atuar na área, pois quando finaliza a graduação ainda não apresenta o domínio necessário que a profissão exige, esta preparação é iniciada nos estágios e aprimorada no dia a dia do trabalho.

Dentre as dificuldades encontradas na realização da pesquisa, percebeu-se que os profissionais relataram a sobrecarga de trabalho, e em algumas unidades aguardamos um tempo maior, para que estes tivessem disponibilidade para preencher o questionário, porém alguns profissionais se abstiveram de participar da pesquisa.

Em relação as limitações deste estudo têm-se a questão de que a informação sobre cuidados paliativos ter sido obtida por entrevista e, portanto, estar sujeita a viés de memória e de informação, o que leva a crer a possibilidade de estudos posteriores nessa temática.

Conclusão

As práticas das ESF estão diretamente relacionadas com os cuidados das pessoas de sua área de abrangência. Têm em geral conhecimento de suas necessidades, quanto ao processo de terminalidade da vida, ofertando ao indivíduo uma interação com seus familiares, bem como proporcionando conforto, alívio da dor e sofrimento, para os pacientes que não tem prognóstico.

Identificamos que os CP já são realizados nas unidades estudadas, porém são encontrados alguns entraves na sua execução como, por exemplo, quanto à comunicação entre o trinômio pessoas-familiares-profissionais de saúde, que pode dificultar a assistência prestada, além da falta de apoio aos envolvidos no processo de adoecimento.

Os trabalhadores da área da enfermagem possuem percepções aguçadas quanto às necessidades que estes indivíduos e suas famílias têm, podendo assim proporcionar dignidade e respeito, para que estes pacientes tenham uma boa morte. É preconizado que o enfermeiro realize a Sistematização da Assistência em Enfermagem, conforme disposto na Resolução do COFEN no 358/2009, que pode beneficiar o cuidado que é prestado à pessoa e família.

Assim, pensando na grandiosidade e complexidade dos CP, o preparo desses funcionários é essencial, bem como um suporte emocional a estes que lidam no dia a dia da sua profissão com o sofrimento e finitude da vida.

Espera-se com este estudo contribuir como alerta para que os profissionais busquem qualificação e aperfeiçoamento para o desenvolvimento dos CP na sua unidade de trabalho, bem como aguçar os gestores para a realização de educação em CP destinados à equipe, além dos setores responsáveis pelas Instituições de Ensino incluírem este tema como disciplina nos cursos de graduação.

\section{Referências}

1. Ministério da Saúde (BR). Coordenação Institucional de Reinaldo Ayer de Oliveira, Conselho Regional de Medicina do Estado de São Paulo. Cuidado Paliativo. São Paulo; 2008. 689 p.

2. Paiva FCL, Almeida Jr JJ, Damásio AC. Ética em cuidados paliativos: concepções sobre o fim da vida. Rev Bioét 2014;22(3). https://doi.org/10.1590/198380422014223038

3. Ministério da Saúde (BR). Academia Nacional de Cuidados Paliativos (ANCP). Manual de cuidados paliativos. $2^{a}$ ed. Brasília: Ministério da Saúde; 2012.

4. Organização Mundial da Saúde (OMS). Who definition of palliative care. [citado 2020 Mar 24]. Disponível: http://www.who.int/cancer/palliative/definition/en/

5. Ministério da Saúde (BR). Secretaria de Atenção à Saúde, Departamento de Atenção Básica. Caderno de atenção domiciliar. Brasília: Ministério da Saúde; 2012;2.

6. Hennemann-Krause L, Freitas LA, Daflon PMN. Cuidados paliativos e medicina de família e comunidade: conceitos e interseções. Rev HUPE 2016;15(3). https://doi.org/10.1590/S1413-81232013000900016

7. Strauss A, Corbin J. Pesquisa qualitativa: técnicas e procedimentos para 0 desenvolvimento de teoria fundamentada. $2^{2}$ ed. Porto Alegre: Artmed; 2008. 
8. Scherer MDA, Oliveira $\mathrm{CI}$, Carvalho WMES, Costa MP. Curso de especialização em Saúde da Família: o que muda no trabalho com a formação? Interface (Botucatu) 2016;20(58). https://doi.org/10.1590/1807-57622015.0020

9. Minayo MCS. Pesquisa social: teoria, método e criatividade. 29aㅡ ed. Petrópolis: Vozes; 2010.

10. IBGE. IBGE cidades. [atualizado 2017; [citado 2017 jul 1]. Disponível: https://cidades.ibge.gov.br/brasil/mg/divinopolis/panorama

11. Bardin L. Análise de conteúdo. Lisboa: Edições 70 Brasil; 2011.

12. Durante ALTC, Tonini T, Armini LR. Conforto em cuidados paliativos: o saber-fazer do enfermeiro no hospital geral. Rev Enferm UFPE online 2014;8(3) [citado 2017 Nov 11]. Disponível: http://studylibpt.com/doc/5342314/conforto-em-cuidados-paliativos--o-saber

13. Santana JCB, Pessini L, Sá AC. Vivências de profissionais da saúde frente ao cuidado de pacientes terminais. Rev Enferm 2017;20(1) [citado 2017 Nov 11]. Disponível: http://periodicos.pucminas.br/index.php/enfermagemrevista/article/view/15410/11790

14. Lima MPO, Oliveira MCX. Significados do cuidado de enfermagem para familiares de pacientes em tratamento paliativo. Rev Rene 2015;16(4). [citado 2017 Nov 11]. Disponível: http://www.redalyc.org/html/3240/324041519017/

15. Rocha AFP, Sposito AMP, Bortoli PS, Silva-Rodrigues FM, Lima RAG, Nascimento LC. O alívio da dor oncológica: estratégias contadas por adolescentes com câncer. Texto Contexto Enferm 2015;24(1). https://doi.org/10.1590/0104-07072015002120013

16. Silveira NR, Nascimento ERP, Rosa LM, Jung W, Martins SR, Fontes MS. Cuidado paliativo e enfermeiros de terapia intensiva: sentimentos que ficam. Rev Bras Enferm 2016;69(6). https://doi.org/10.1590/0034-7167-2016-0267

17. Alves RF, Andrade SFO, Melo MO, Cavalcante KB, Angelim RM. Cuidados paliativos: desafios para cuidadores e profissionais de saúde. Rev Psicol 2015;27(2). https://doi.org/10.1590/1984-0292/943

18. Conselho Federal de Enfermagem. Resolução n.358, de 15 de outubro de 2009. Dispõe sobre a Sistematização da Assistência de Enfermagem e a implementação do Processo de Enfermagem em ambientes, públicos ou privados, em que ocorre o cuidado profissional de Enfermagem, e dá outras providências. Brasília: COFEN; 2009. [citado 2017 Dez 13]. http://www.cofen.gov.br/resoluo-cofen-3582009 4384.html

19. Instituto Nacional de Câncer [Internet]. Rio de Janeiro: Instituto Nacional de Câncer José Alencar Gomes da Silva. [citado 2017 Nov 11]. Disponível: http://www2.inca.gov.br/wps/wcm/connect/cancer/site/tratamento/cuidados paliativos

20. Góis ARS, Abrão FMS. O processo de cuidar do enfermeiro diante da morte. Rev Enferm UFSM 2015;(3). https://doi.org/10.5902/2179769215832

21. Silva MM, Santanda NGM, Santos MC, Cirilo JD, Barrocas DLR, Moreira MC. Cuidados paliativos na assistência de alta complexidade em oncologia: percepção de enfermeiros. Rev Esc Anna Nery 2015;19(3). https://doi.org/10.5935/1414$\underline{8145.20150061}$ 